\title{
Flexible and Transparent Surface-Enhanced Raman Scattering (SERS)-Active Metafilm for Visualizing Trace Molecules via Raman Spectral Mapping
}

\author{
Xiangjiang Liu, ${ }^{\dagger}$ Jingjing Wang, ${ }^{\dagger}$ Jiajun Wang, Longhua Tang, ${ }^{*} *$ and Yibin Ying ${ }^{\dagger}$ \\ ${ }^{\dagger}$ College of Biosystems Engineering and Food Science, Zhejiang University, Hangzhou 310058, \\ China. \\ ¥ State Key Laboratory of Modern Optical Instrumentation, College of Optical Science and \\ Engineering, Zhejiang University, Hangzhou 310027, China \\ * Corresponding author: Email: 1htang@,zju.edu.cn (L. H Tang)
}

RECEIVED DATE (to be automatically inserted after your manuscript is accepted if required according to the journal that you are submitting your paper to) 


\section{Materials and Instruments}

Gold (III) chloride hydrate $\left(\mathrm{HAuCl}_{4}\right)$, hexadecyltrimethylammonium bromide (CTAB), cetyltrimethylammonium chloride (CTAC), thiolated poly(ethylene glycol) (average M.W. 5000, PEG-SH), sodium borohydride $\left(\mathrm{NaBH}_{4}\right)$, sodium citrate, $p$-aminothophenol (PATP), crystal violet (CV), Rhodamine B (RB), hexadecanethiol were purchased from Sigma-Aldrich (Shanghai). Silver nitrate $\left(\mathrm{AgNO}_{3}\right)$ was obtained from Amersco Co. Sylgard 184 silicone elastomer base and Sylgard 184 elastomer curing agent were obtained from Dow Corning. The above reagents were used without further purification and Milli-Q water $\left(18 \mathrm{M} \Omega \cdot \mathrm{cm}^{-1}\right)$ was used to prepare all aqueous solutions.

A LabRAM HR Evolution Raman microscope system (Horiba Jobin Yvon, Kyoto) equipped with an integral Olympus BX40 microscope and an automatic motorized XYZ stage was used to for all the SERS measurements. Extinction spectra were measured using a UV/vis spectrophotometer (Evolution 300, Thermos Fisher, USA). Fluorescent images were taken by an inverted fluorescence microscope (Nikon Eclipse Ti). SEM images were collected by a field emission scanning electron microscopy (Zeiss Utral-55).

\section{Fabrication of PEG-SH capped AgNCs.}

Silver nanocubes (AgNCs) were prepared according using the previous reported seed-mediated growth method. ${ }^{1}$ Briefly, CTAB-capped Au seeds $(\sim 3 \mathrm{~nm})$ were prepared by mixing $0.6 \mathrm{~mL}$ of 10

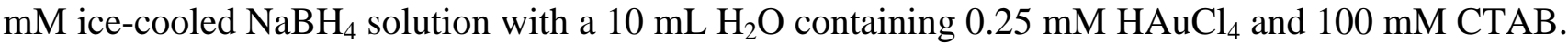
The seed solution was kept undisturbed for $3 \mathrm{~h}$ at $27^{\circ} \mathrm{C}$. Then CTAC-capped $\mathrm{Au}$ seeds $(\sim 11 \mathrm{~nm})$ were synthesized by adding $4.5 \mathrm{~mL}$ of $100 \mathrm{mM}$ AA solution into a beaker containing $0.3 \mathrm{~mL}$ of the 3 $\mathrm{nm}$ Au seeds, $6 \mathrm{~mL}$ of $0.5 \mathrm{mM} \mathrm{HAuCl}{ }_{4}$ and $6 \mathrm{~mL}$ of $200 \mathrm{mM} \mathrm{CTAC}$. The CTAC-capped Au seeds were collected by centrifugation and washed with $\mathrm{H}_{2} \mathrm{O}(14,500 \mathrm{rpm}, 10 \mathrm{~min})$ once before used for 
AgNC preparation. Then, $20 \mathrm{~mL}$ of aqueous solution of $50 \mathrm{mM}$ AA and $40 \mathrm{mM}$ CTAC and $20 \mathrm{~mL}$ of $2 \mathrm{mM} \mathrm{AgNO} 3$ were simultaneously injected $(0.8 \mathrm{~mL} / \mathrm{min})$ into a $100 \mathrm{~mL}$ vial containing $2 \mathrm{~mL}$ of the CTAC-Au seeds and $18 \mathrm{~mL}$ of $20 \mathrm{mM} \mathrm{CTAC}$. The mixture was kept at $60{ }^{\circ} \mathrm{C}$ for $4 \mathrm{~h}$ and was cooled in an ice bath. The resulting CTAC-capped AgNCs were collected by centrifugation (12,000 rpm, 15 min) and redispersed in $20 \mathrm{~mL}$ of water for further use.

The obtained CTAC-capped AgNCs were transfer from aqueous solution to chloroform $\left(\mathrm{CHCl}_{3}\right)$ according previous described procedure with slight modification. ${ }^{2}$ Briefly, $5 \mathrm{~mL}$ of CTAC-capped AgNCs aqueous was mixed with $5 \mathrm{~mL} \mathrm{CHCl}_{3}$ containing $0.1 \mathrm{mg} / \mathrm{mL}$ PEG-SH. Upon addition of 1 $\mathrm{mL}$ methanol to the aqueous phase, the AgNCs were quickly transfer to the chloroform layer. The resulting PEG-SH capped AgNCs were collected by centrifugation (8000 rpm for 10min) and washed with $\mathrm{CHCl}_{3}$ twice, followed by redispersion in $\sim 50 \mu \mathrm{L}$ chloroform. 


\section{Fabrication of the metafilm}

a
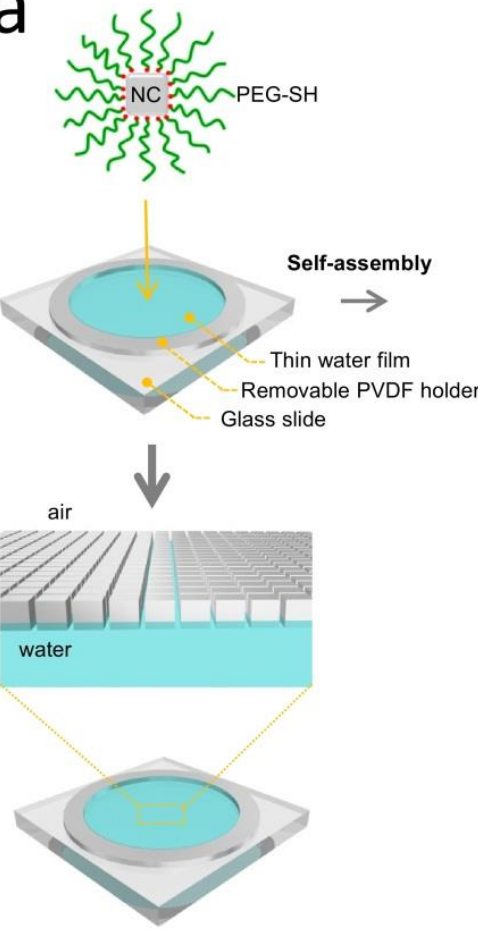

12 Drained

Remove holder

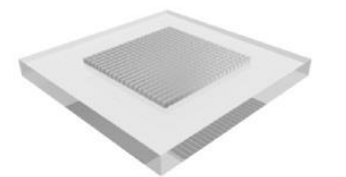

NC arrays on glass

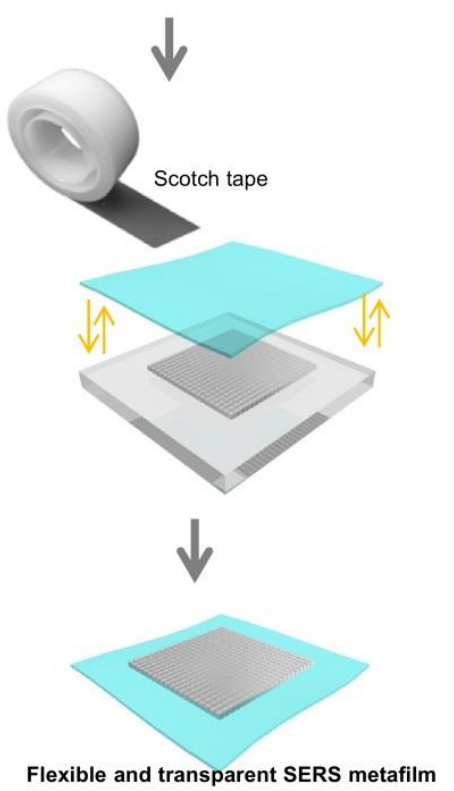

b

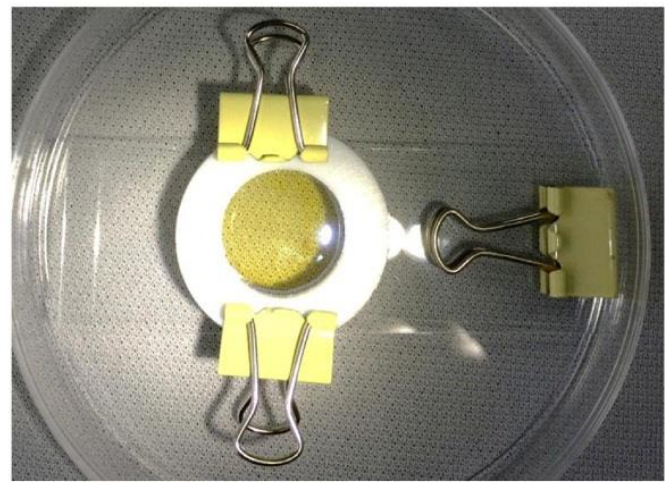

C
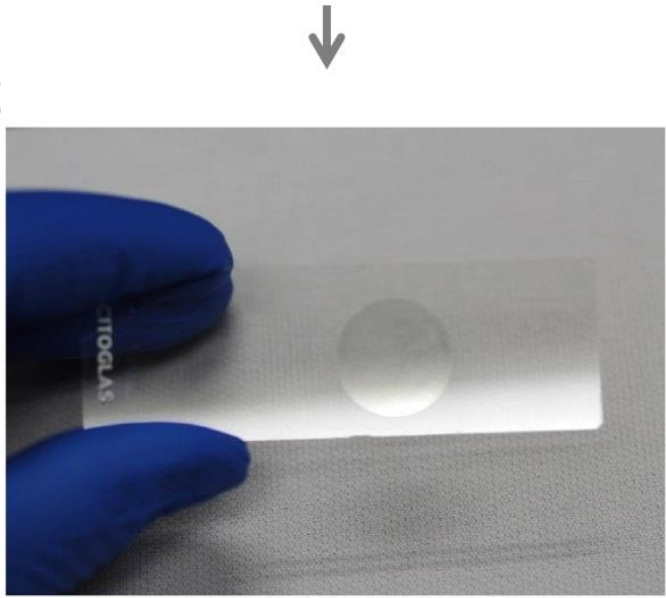

$\downarrow$

d
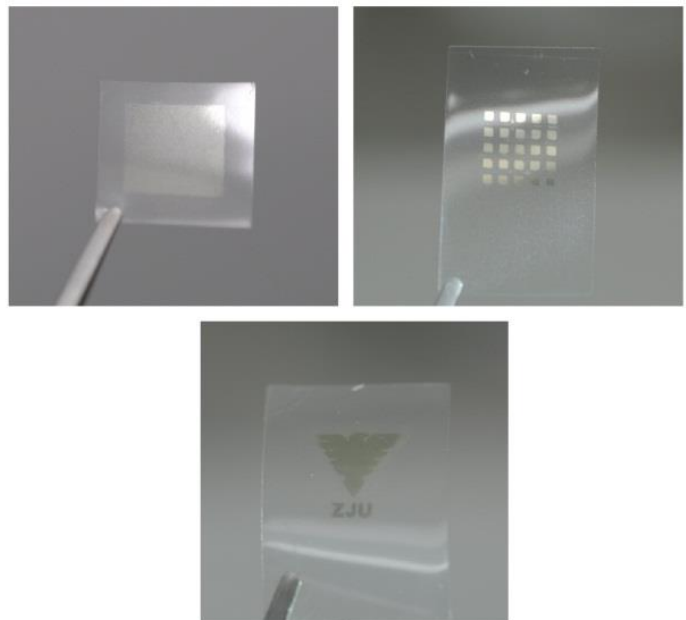

Figure S1 (a) Schematic illustration of the fabrication approach; (b) a simple setup developed for 
LLI self-assembly operation. A thin PVDF holder was placed on a glass slide to confine the thin water film and prevented the formed superlattice from breaking and moving around on the glass; (c) the resulting AgNCs superlattice sheet on a glass slide; (d) the AgNCs superlattice sheets picked up by the Scotch tapes. When patterned AgNCs superlattice are wanted, it can be firstly treated with an adhesive tape with pre-drilled holes and then the remaining patterned AgNCs superlattice can be picked up by the tape

\section{Samples preparation.}

\section{a. Preparation of the PDMS stamp.}

The Silica master fabricated by photolithography technique was obtained from Wenhao Chip Co. (Shuzhou, China). The PDMS stamp was fabricated by casting a liquid PDMS precursor (Sylgard 184) against the master. In brief, 10 parts of Sylgard 184 base was thoroughly mixed with 1 part of the curing agent by weight, and was poured on the silica master placed in a Petri dish. After the trapped air bubbles in the precursor were removed by the vacuum, it was cured at $120{ }^{\circ} \mathrm{C}$ for $1 \mathrm{~h}$. The resulting PDMS stamp was carefully peeled off from the mater using a razor blade.
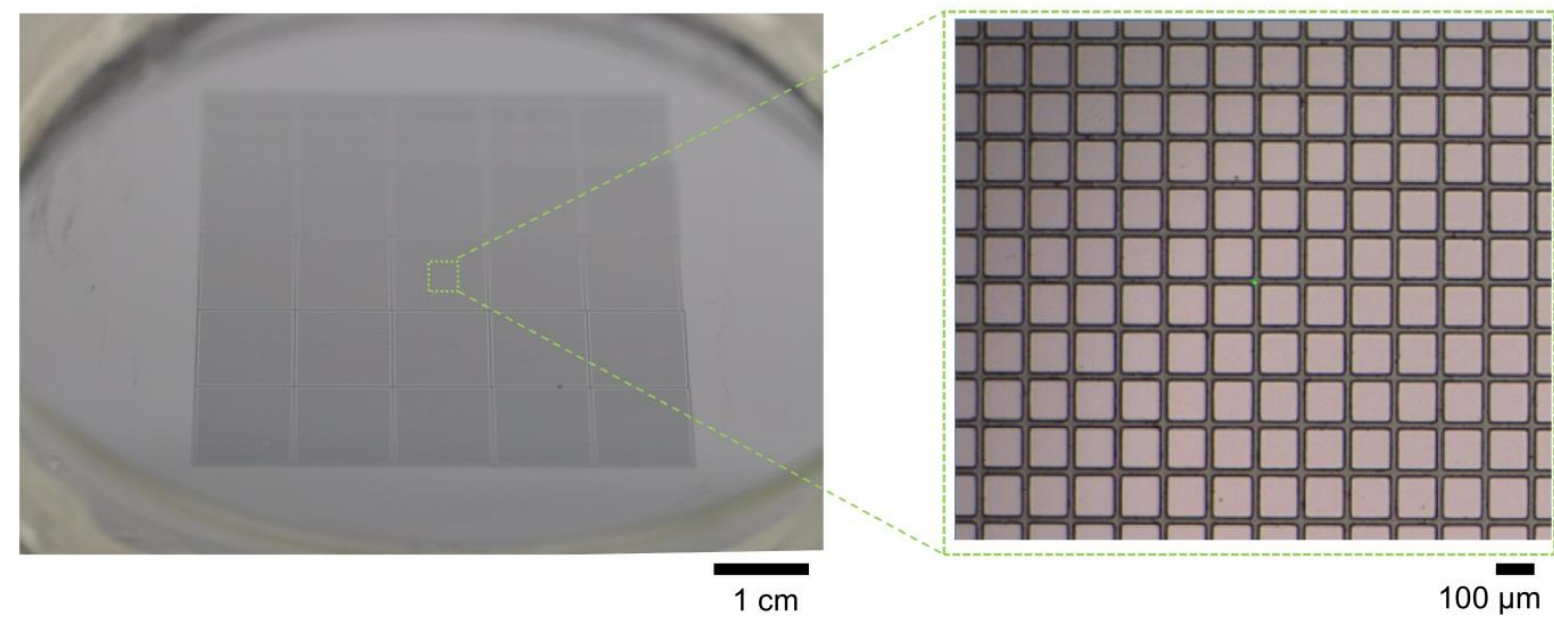

Figure S2 Optical images of the obtained silica master. 


\section{b. Pattered RB array on the glass slide.}

The SAMs Raman probe patterns were fabricated by the microcontact printing approach. ${ }^{3}$ Briefly, a PDMS stamp containing a periodic $100 \times 100 \mu \mathrm{m}$ squares array with a $20 \mu \mathrm{m}$ gap (see Figure $2 \mathrm{a}$ ), was used to print the dye of Rhodamine B (RB) on the glass slide. Before printing, the glass slides were cleaned in a ultrasonic bath with $\mathrm{H}_{2} \mathrm{O}$, ethanol and acetone, and dried in a $\mathrm{N}_{2}$ flow. Then, small amount of $10^{-3} \mathrm{M}$ RB ethanol solution was swabbed on the PDMS stamp with a cotton Q-tip for $5 \mathrm{~s}$ and dried in a gentle $\mathrm{N}_{2}$ flow for $30 \mathrm{~s}$. Next, the PDMS stamp was carefully attached to the cleaned glass slide about $10 \mathrm{~s}$. It is particularly important to avoid trapping any air bubbles between the stamp and the slide in this step. The PDMS stamp was quicky separated from the substrate (without dragging), and the reulting patterned RB array was dired for by a gentle $\mathrm{N}_{2}$ flow for 10 s. Then, a SERS-active metafilm (face down) was firmly attached to the patterned RB array with the assistance of a rubber roller, before Raman mapping.

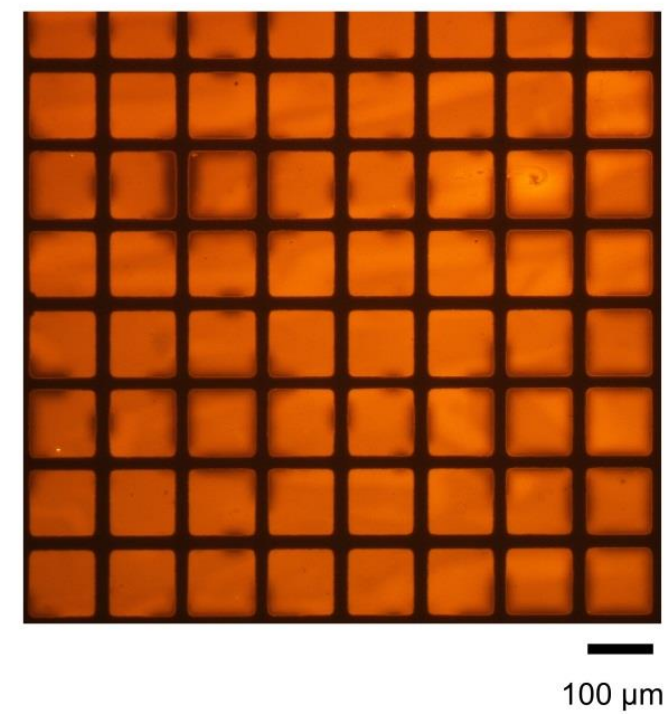

Figure S3 Typical fluorescence image of the resulting pattered RB array of the glass slide. 


\section{PATP SAMs on a patterned gold surface.}

The gold substrate was prepared by depositing a conventional silica wafer with a $50 \mathrm{~nm}$ gold film using a sputter coater (Quorum Q150R ES) at a rate of $\sim 0.8 \AA \cdot \mathrm{s}^{-1}$ under vacuum $\left(3 \times 10^{-2} \mathrm{mbar}\right)$. An adhesion layer of $5 \mathrm{~nm}$ titanium was despoted between the gold and the silica wafer in advance. Then, small amount of $2 \mathrm{mM}$ hexadecanethiol (HDT) ethanol solution was swabbed on the PDMS stamp for $5 \mathrm{~s}$ and dried in $\mathrm{N}_{2}$ flow. Then, the PDMS stamp was filmy contacted with the gold substrate for $10 \mathrm{~s}$ and quickly separated afterwards. Afterwards, the obtained gold substrate with patterned HDT monolayers was immersed the obtained in a fresh-prepared ferricyanide etching solution $\left(0.1 \mathrm{M} \mathrm{K}_{2} \mathrm{~S}_{2} \mathrm{O}_{3}, 0.01 \mathrm{M} \mathrm{K}_{3} \mathrm{Fe}(\mathrm{CN})_{6}, 0.001 \mathrm{M} \mathrm{K}_{4} \mathrm{Fe}(\mathrm{CN})_{6}\right.$ and $\left.1 \mathrm{M} \mathrm{KOH}\right)$ for several minutes at room temperature, which could selectively remove the unprotected region of gold substrate. The resulting pattered gold substrate was treated with $0.1 \mathrm{~mW}$ PATP solution for $1 \mathrm{~h}$ and a PATP SAM on patterned gold surface was obtained. After rinsed by water and dried by $\mathrm{N}_{2}$ flow, the patterned SAM of PATP was firmly attached to a SERS-active metafilm placed on before measurement.

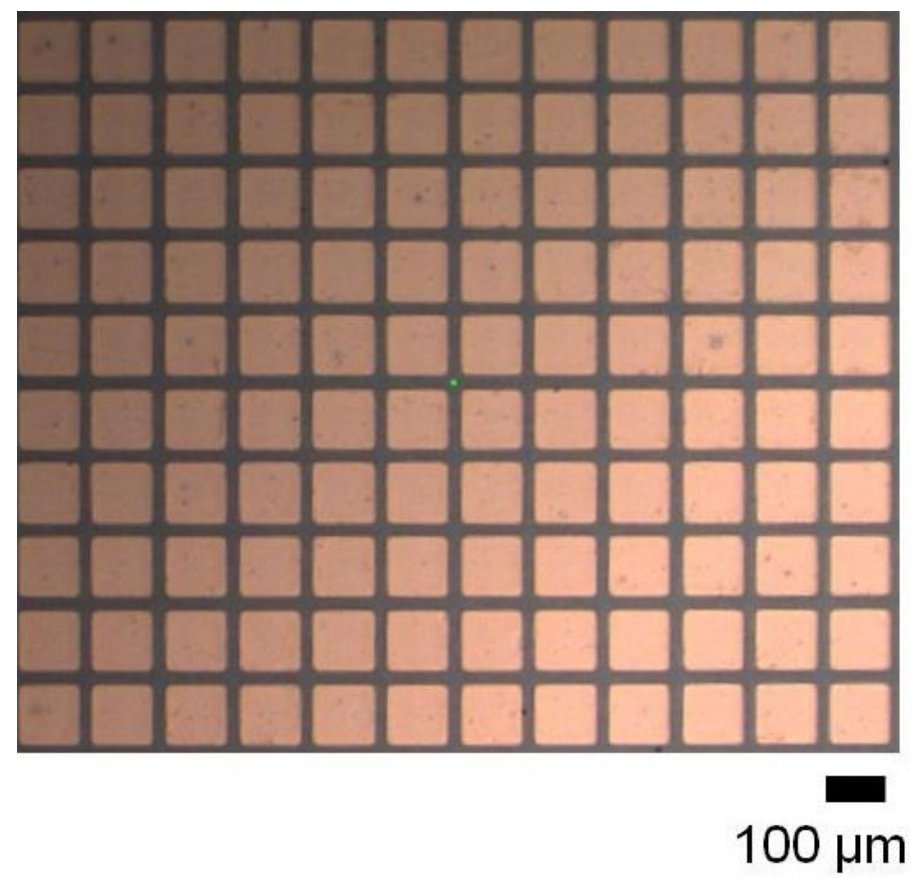

Figure S4 Typical microscopy image of the resulting the PATP SAMs on a patterned gold surface. 
IV. Characterizations.

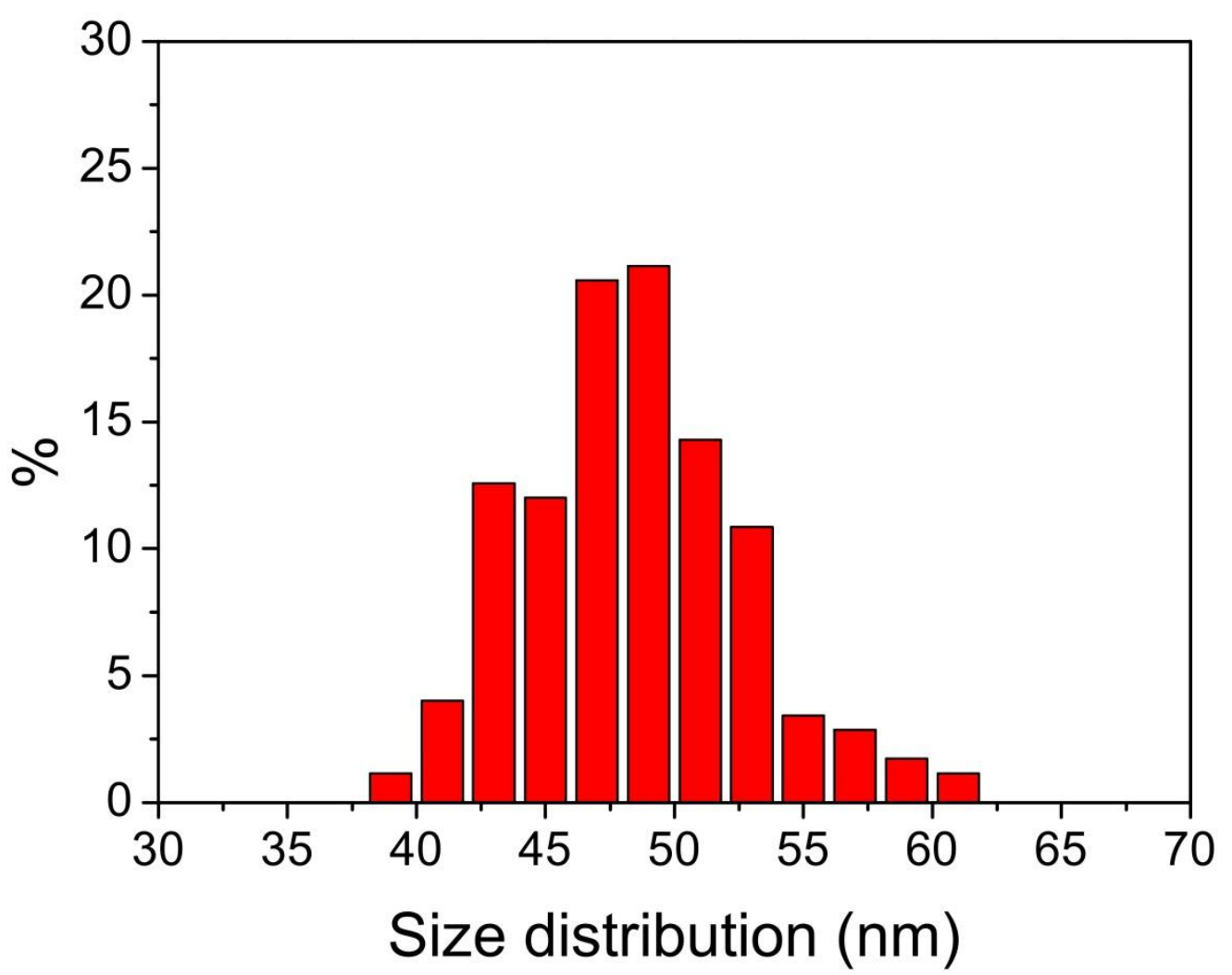

Figure S5 Size distribution of as-prepared AgNCs (a total of 175 particles were analyzed) 
a

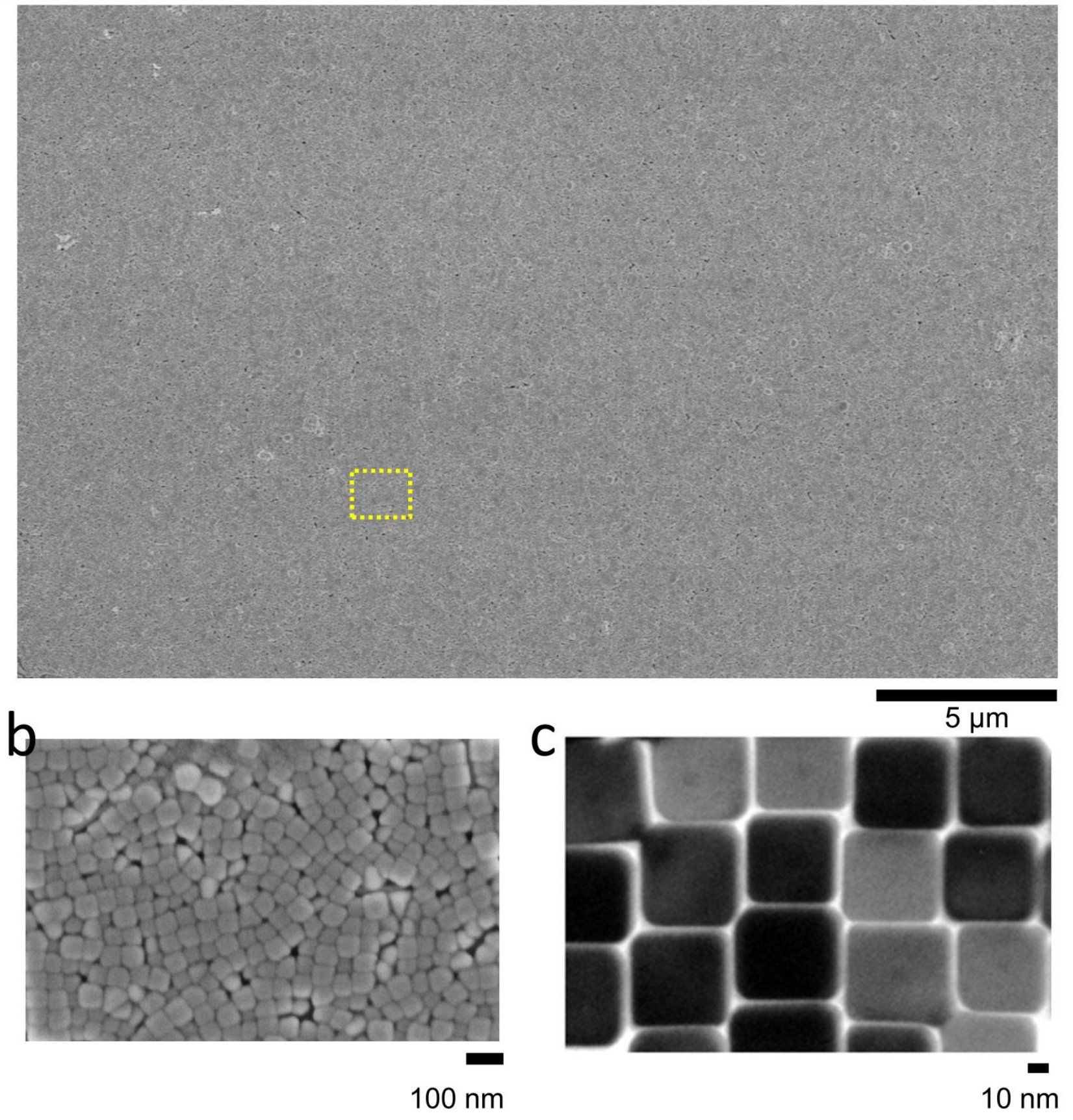

Figure S6 (a, b) SEM and (C) TEM images of AgNCs superlattice sheet. 


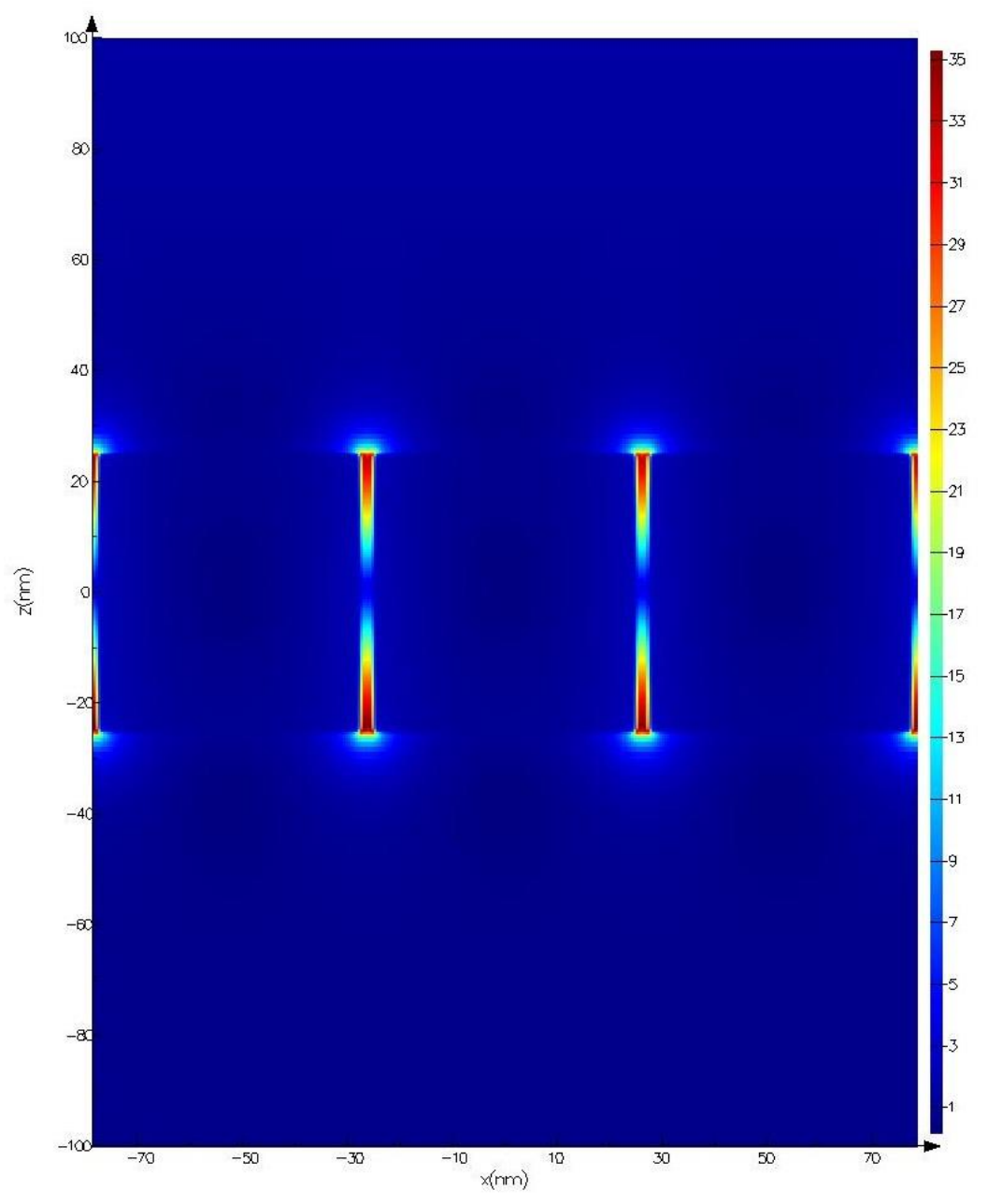

Figure S7 A FDTD simulation of the local electric field enhancement for the AgNCs superlattice (AgNCs: $50 \mathrm{~nm}$, gap: $2.5 \mathrm{~nm}$ ), which was performed using the commercial modeling package Lumerical solutions. Periodic boundary conditions (for $\mathrm{x}$, y-axis) and PML (for $\mathrm{z}$-axis) were employed and a plane wave source was used in the simulations. The optical constant of sliver was provided by the software package. 


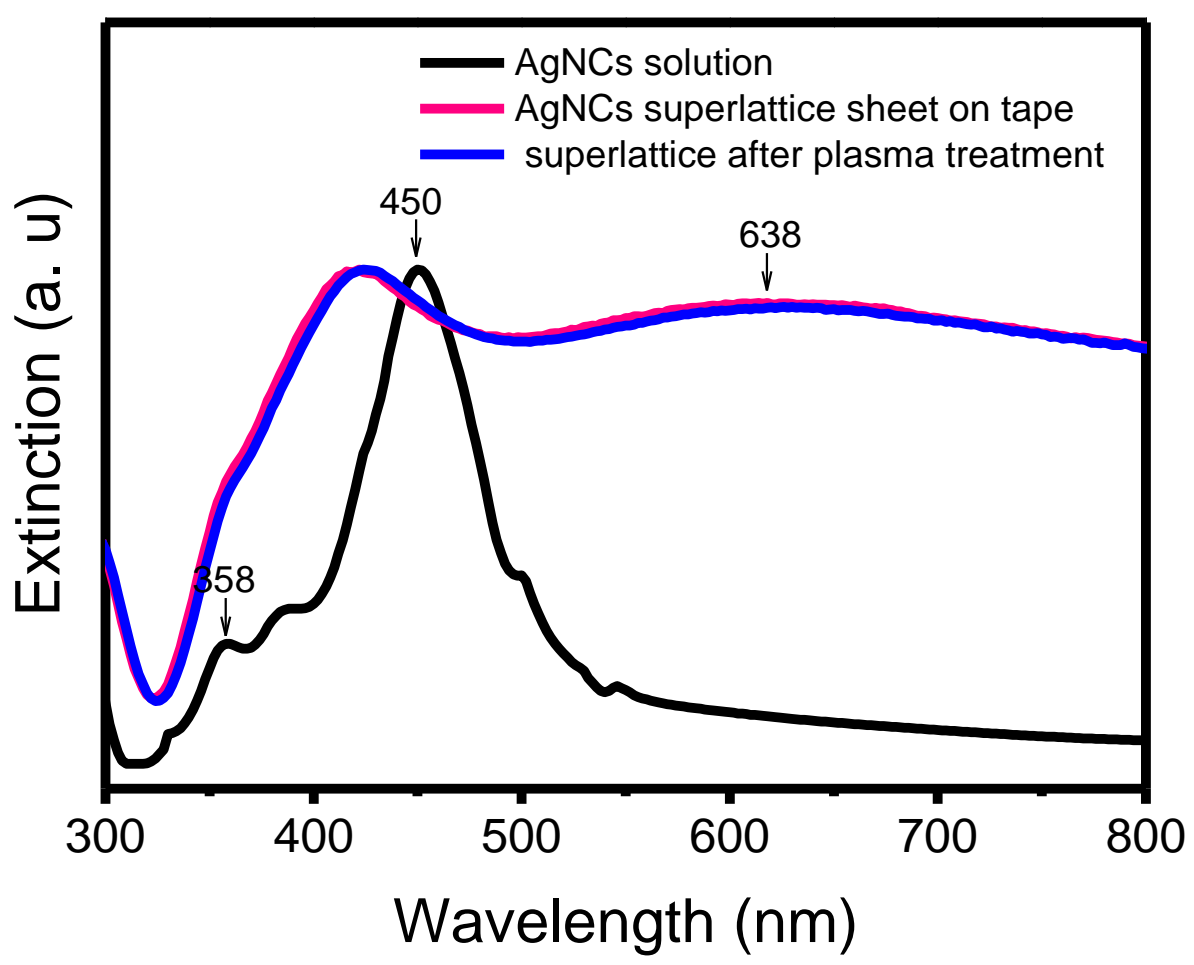

Figure S8. The experimental extinction spectra of FTSM and AgNCs colloidal solution. The resonance peak of metafilm showed slightly red-shift after removing the PEG-SH by O2 plasma. The SERS metafilm displayed a "peak" at $420 \mathrm{~nm}$, which we believe it was caused by the Scotch tape, since the optical transmittance of the tape abruptly around this range (see Figure S13). Such "peak" was not observed for the AgNCs superlattice sheet supported by a glass slide. 


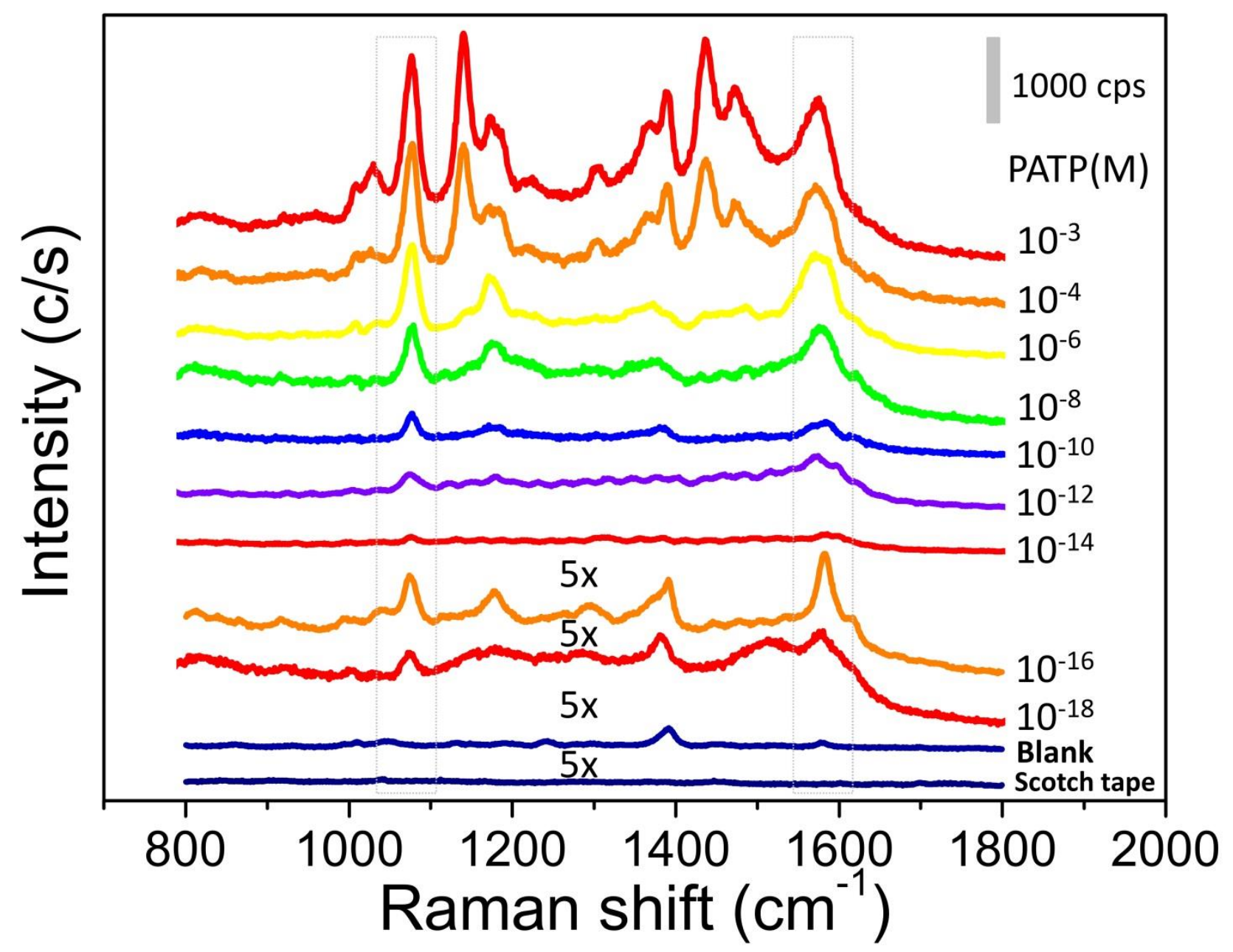

Figure S9 SERS spectra of the FTSM treated with different concentration of PATP. The FTSMs were immersed in a centrifuge tube containing $50 \mathrm{~mL}$ PATP solution with different concentrations. After gently shaking for $1 \mathrm{~h}$, the FTSMs were rinsed and dried by $\mathrm{N}_{2}$ gas flow and measured by our spectroscopy. (laser power $0.85 \mathrm{~mW}$; typical integration time is $5 \mathrm{~s}$; for low PATP concentration $\left(10^{-12}\right.$ to $\left.10^{-18} \mathrm{M}\right)$, integration time $10 \mathrm{~s}$, averaging 5 times). 


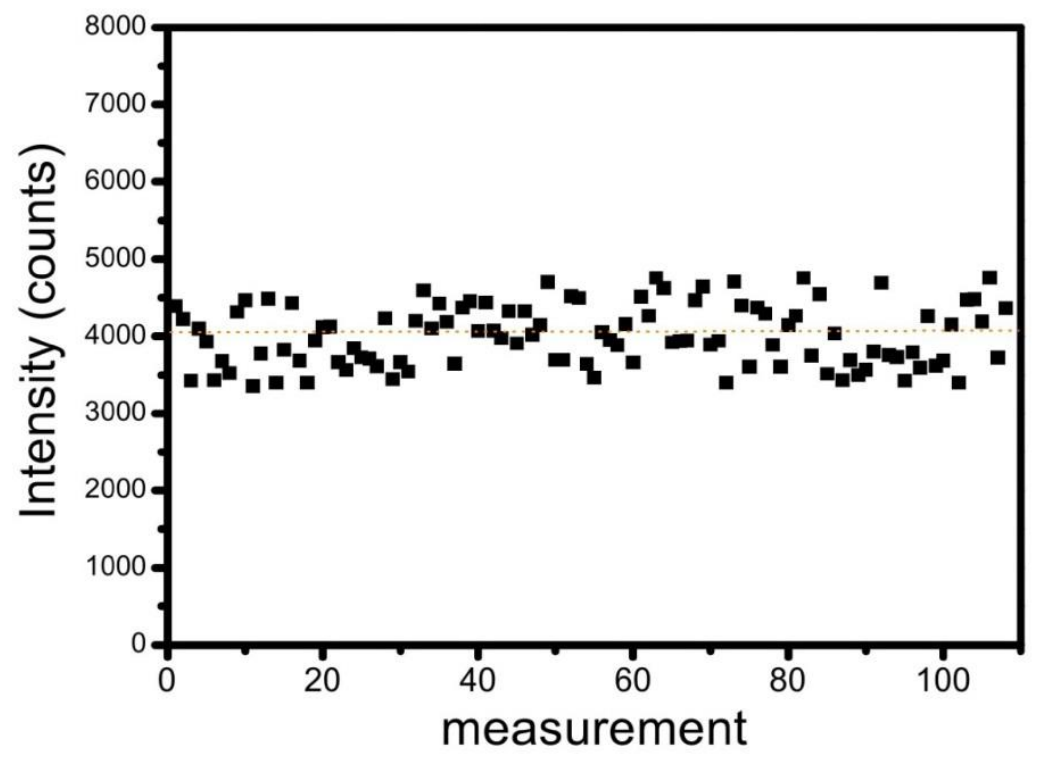

Figure S10 SERS intensity distribution of the FTSM (laser power $0.85 \mathrm{~mW}$; integration time $10 \mathrm{~s}$ ).

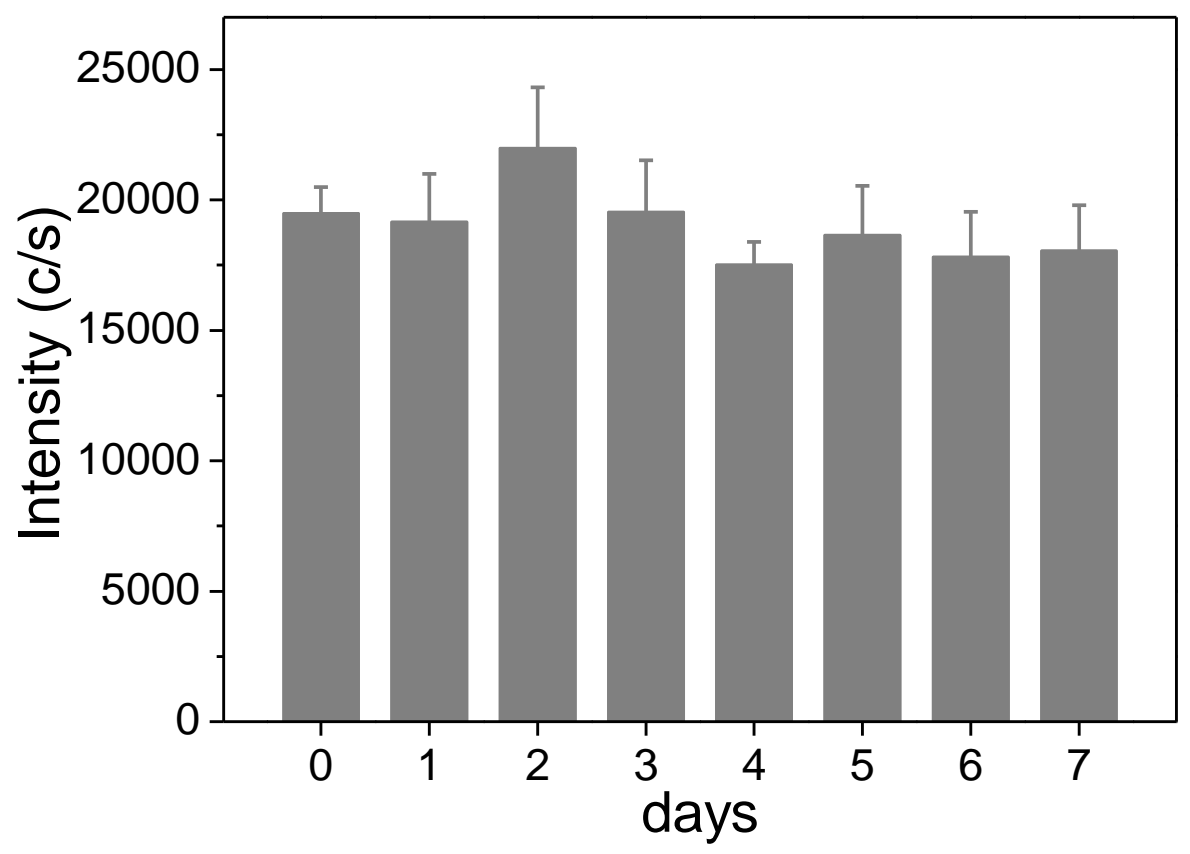

Figure S11 SERS activities of AgNCs-superlattice sheet on the glass slide stored in a stored in a desiccator (at $1617 \mathrm{~cm}^{-1}$, treated with $10^{-5} \mathrm{M} \mathrm{CV}$ ). 


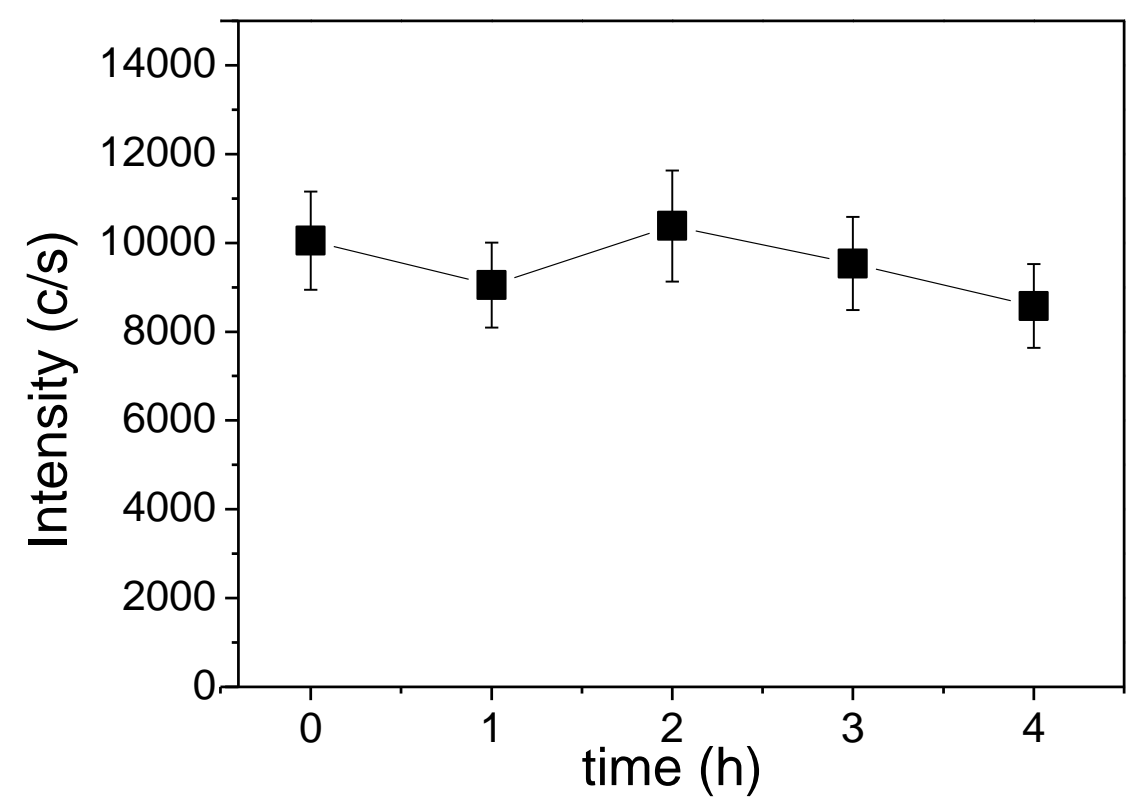

Figure S12 SERS activities of an FTSM during a measurement. (1617 $\mathrm{cm}^{-1}$ of CV)

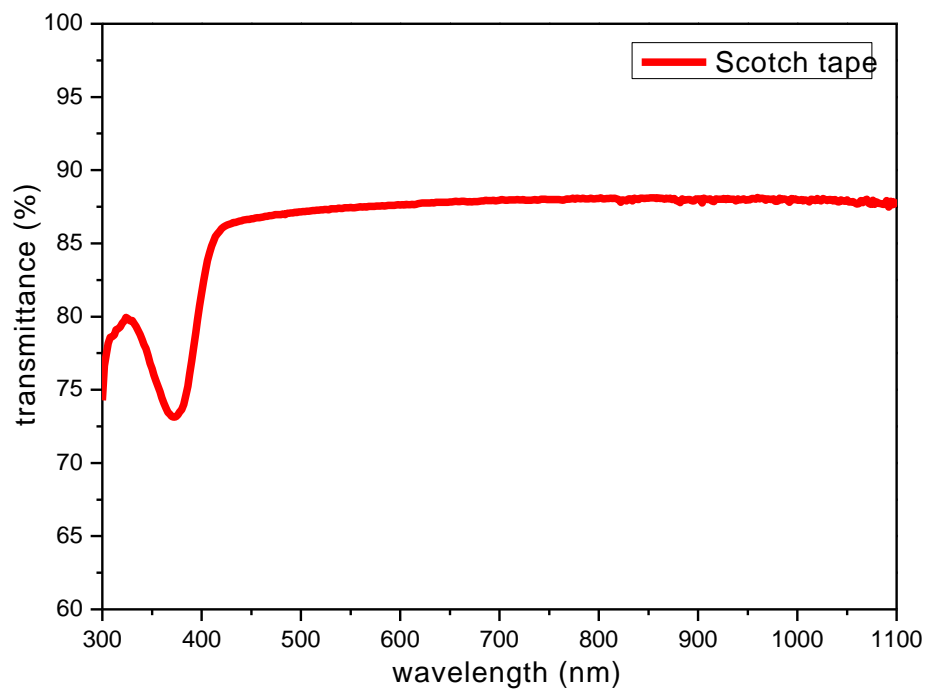

Figure S13 Transmittance spectrum of the Scotch tape. 


\section{SERS Enhancement Factors (EFs)}

The SERS EF can be calculated using the following equation:

$$
\mathrm{EF}=\frac{I_{S E R S} \cdot N_{B u l k}}{I_{B u l k} \cdot N_{S E R S}}
$$

where $I_{S E R S}$ and $I_{B u l k}$ are the same band for SERS and bulk spectra. $N_{S E R S}$ and $N_{B u l k}$ refer to the number of PATP molecules in the illumination area. The detailed calculation is shown below.

(1) We assumed that PATP molecules were adsorbed as a complete monolayer with a molecular footprint of $\sigma=0.2 \mathrm{~nm}^{2}$. With the radius of laser spot $\mathrm{R}_{0}=0.61 \lambda / \mathrm{NA}=0.77 \mu \mathrm{m}(50 \mathrm{x}$, numerical aperture $0.5,633 \mathrm{~nm}$ ), and the PATP molecule footprint size, $N_{S E R S}=\pi \mathrm{R}_{0}{ }^{2} / \sigma=\sim 2.9 \times 10^{6}$

(2) The effective focal volume is approximated by following equation $V_{\text {focal }}=\pi^{3 / 2} R_{0}^{2} Z_{0}$, where the depth of focus $\mathrm{Z}_{0}=2 \pi \mathrm{R}_{0}^{2} / \lambda$. Therefore, the $\mathrm{V}_{\text {focal }}$ is calculated to be $1.97 \times 10^{-14} \mathrm{~L}$.

(3) $N_{b u l k}$ is the number of PATP molecules within the effective focal volume of a bulk sample. $N_{b u l k=}$ $\mathrm{V}_{\text {focal }} \rho / M W \times 6.02 \times 10^{23}$, where the density of PATP $\rho$ is $1.2 \mathrm{~g} / \mathrm{cm}^{3}$ and the molecular weight $M W$ is $125.19 \mathrm{~g} / \mathrm{mol} . N_{\text {bulk }}$ is determined to be $1.14 \times 10^{11}$

(4) The areas of the $\sim 1076 \mathrm{~cm}^{-1}$ band were used for the intensities $I_{\text {SERS }}(41197.6)$ and $I_{\text {Bulk }}(498.06)$, which were obtained from experiments.

(5) $\mathrm{EF}=\sim 3.2 \times 10^{6}$ 


\section{Additional experimental results.}

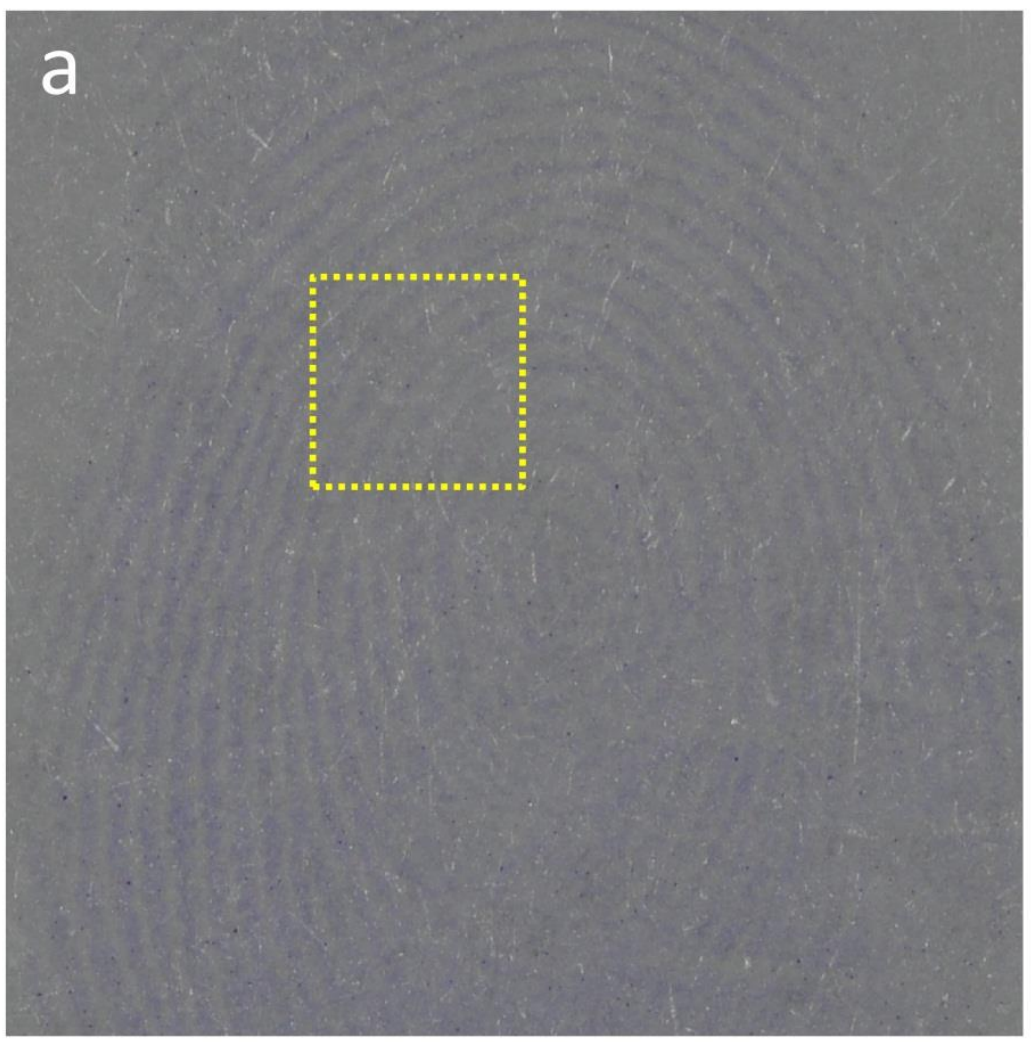

b

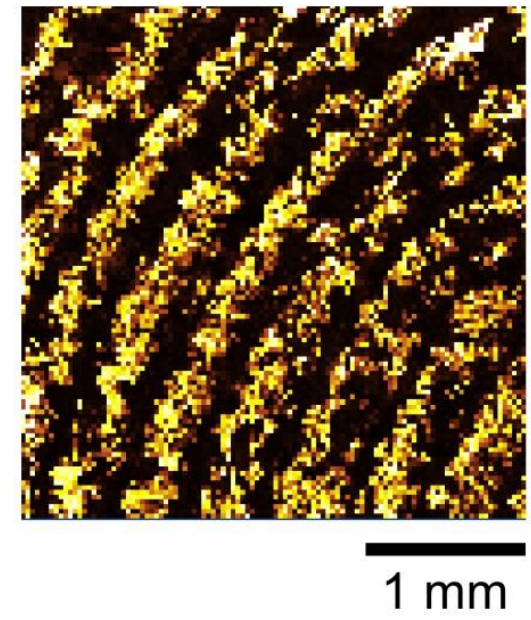

Figure S14 (a) a visible fingerprint on a print paper and (b) its corresponding SERS mapping image.
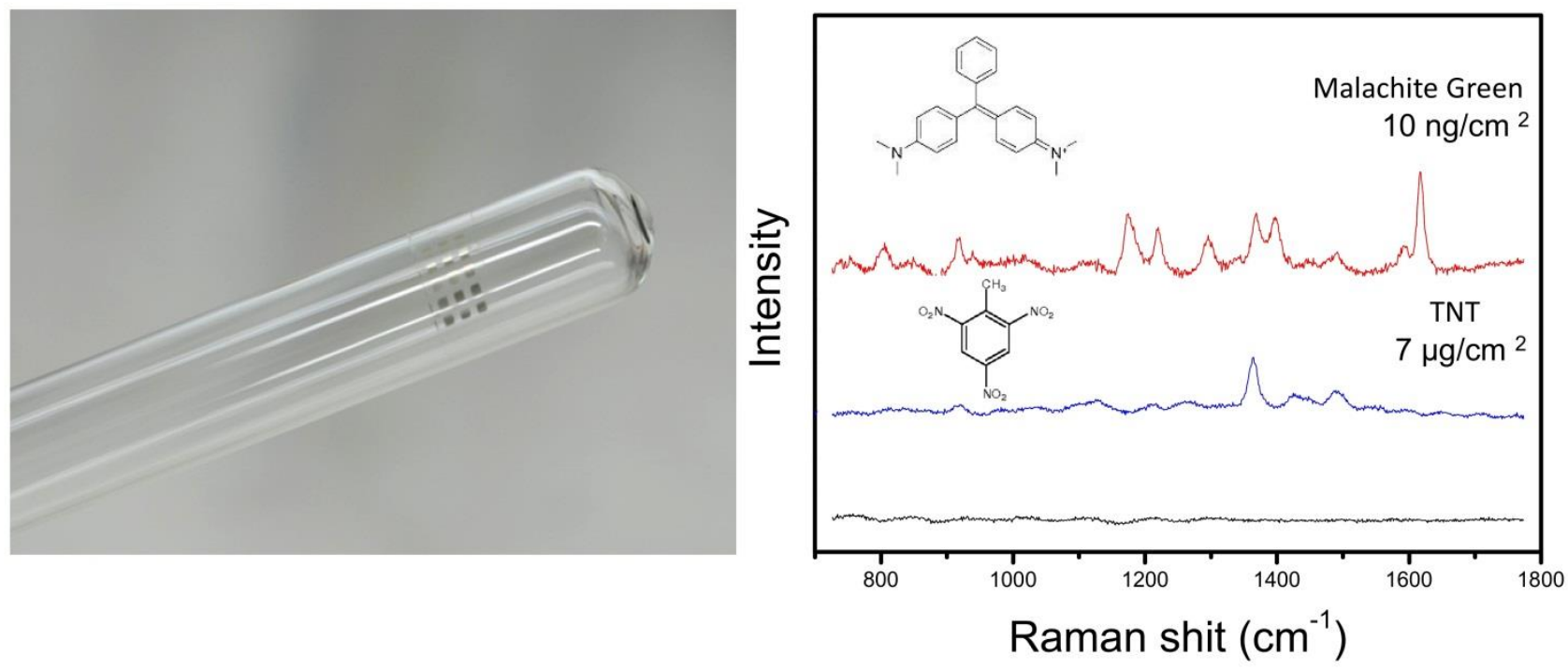

Figure S15 Detection TNT residue and malachite residue on a glass tube. 


\section{Reference}

1. Palonpon, A. F.; Ando, J.; Yamakoshi, H.; Dodo, K.; Sodeoka, M.; Kawata, S.; Fujita, K. Nat. Protocols 2013, 8 , 677-692.

2. Alkilany, A. M.; Yaseen, A. I. B.; Park, J.; Eller, J. R.; Murphy, C. J. RSC Adv. 2014, 4, 52676-52679.

3. Qin, D.; Xia, Y.; Whitesides, G. M. Nat. Protocols 2010, 5, 491-502. 